\title{
Voxel-based meta-analysis of grey matter changes in Alzheimer's disease
}

\author{
Wen-Ying Wang ${ }^{1}$, Jin-Tai Yu ${ }^{1,2,3^{*}}$, Yong Liu ${ }^{4,5}$, Rui-Hua Yin ${ }^{1}$, Hui-Fu Wang ${ }^{3}$, Jun Wang ${ }^{1}$, Lin Tan², Joaquim Radua ${ }^{6,7}$ \\ and Lan $\operatorname{Tan}^{1,2,3^{*}}$
}

\begin{abstract}
Background: Voxel-based morphometry (VBM) using structural brain MRI has been widely used for the assessment of impairment in Alzheimer's disease (AD), but previous studies in VBM studies on AD remain inconsistent.

Objective: We conducted meta-analyses to integrate the reported studies to determine the consistent grey matter alterations in AD based on VBM method.

Methods: The PubMed, ISI Web of Science, EMBASE and Medline database were searched for articles between 1995 and June 2014. Manual searches were also conducted, and authors of studies were contacted for additional data. Coordinates were extracted from clusters with significant grey matter difference between AD patients and healthy controls $(\mathrm{HC})$. Meta-analysis was performed using a new improved voxel-based meta-analytic method, Effect Size Signed Differential Mapping (ES-SDM).

Results: Thirty data-sets comprising 960 subjects with AD and $1195 \mathrm{HC}$ met inclusion criteria. Grey matter volume (GMV) reduction at 334 coordinates in $A D$ and no GMV increase were found in the current meta-analysis. Significant reductions in GMV were robustly localized in the limbic regions (left parahippocampl gyrus and left posterior cingulate gyrus). In addition, there were GM decreases in right fusiform gyrus and right superior frontal gyrus. The findings remain largely unchanged in the jackknife sensitivity analyses.
\end{abstract}

Conclusions: Our meta-analysis clearly identified GMV atrophy in AD. These findings confirm that the most prominent and replicable structural abnormalities in AD are in the limbic regions and contributes to the understanding of pathophysiology underlying AD.

Keywords: Voxel-based morphometry (VBM), Alzheimer's disease (AD), Grey matter (GM), Meta-analysis, Magnetic resonance imaging (MRI), Effect size signed differential mapping (ES-SDM)

\section{Introduction}

Morphometric MRI studies have investigated focal structural abnormalities in brain tissue types, such as grey matter (GM) and white matter (WM), between groups of individuals using voxel-based morphometry (VBM). Briefly, the VBM approach presents lots of advantages such as fully automated, hypothesis-free, time-efficient, operator-independent and capable of investigating grey matter abnormalities across the whole brain than region of interest (ROI) analysis [1].

\footnotetext{
* Correspondence: yu-jintai@163.com; dr.tanlan@163.com

'Department of Neurology, Qingdao Municipal Hospital, School of Medicine, Qingdao University, No.5 Donghai Middle Road, Qingdao, Shandong Province 266071, China

Full list of author information is available at the end of the article
}

However, the major limitation of ROI-based techniques of morphometric brain changes is that this method requires a priori decision concerning which structures need to be evaluated [2]. Due to the small and heterogeneous samples of participants as well as substantial methodological differences between studies, results from VBM studies remain inconsistent and controversial [3]. As an intrinsical whole-brain technique, the VBM method exhibits comparable accuracy to manual volumetry and overcomes the limitations of ROI approach; therefore identifying consistent results from VBM studies of grey matter volume (GMV) in Alzheimer's disease (AD) patients through meta-analysis is of particular significance. 
$\mathrm{AD}$ is the most common type of dementia, the progressive neurodegenerative disorder, characterized by extensive neuronal and synaptic losses, as well as the presence of extracellular amyloid plaques, intracellular neurofibrillary tangles (NFTs) and brain volume reduction $[4,5]$. However, VBM studies of GMV in AD yield variable and conflicting evidence supporting these models; for example, some studies find regional grey matter atrophy mainly restricted to the medial temporal structures including bilateral hippocampus, amygdala and entorhinal cortex, as well as the posterior cingulate gyrus and medial thalamus [6,7], whereas a study found GM loss only in temporoparietal cortex [8].

Signed Differential Mapping (SDM) is a recentlydeveloped statistical technique, which adopts and combines various positive features of activation likelihood estimate (ALE) and multilevel kernel density analysis (MKDA), in order to quantify the reproducibility of neuroimaging findings and generate insights difficult to observe in isolated studies [9]. Therefore, in the present study, we conducted a voxel-wisely meta-analysis VBM studies on AD using an SDM software to identify the consistent regional grey matter abnormalities in AD.

\section{Methods}

\section{Search strategy}

Systematic and comprehensive searches were conducted in PubMed (http://www.ncbi.nlm.nih.gov/pubmed/), ISI Web of Science (www.isiknowledge.com), Embase (www. embase.com/), and Medline databases (http://www. medline.com/) from 1995 to 25 June 2014 using the keywords "Alzheimer's disease" OR "AD”, AND "voxel"”, "morphometry",OR "vbm". A hand searching was also performed in the reference lists of inclusion articles. The studies were considered for inclusion if they (1) reported VBM (GM density or volume) comparison between patients with $\mathrm{AD}$ and $\mathrm{HC}$ subjects; (2) reported whole brain results of changes in a stereotactic space in three dimensional coordinates ( $\mathrm{x}, \mathrm{y}, \mathrm{z})$; (3) used significance thresholds either corrected for voxel based multiple comparisons or uncorrected with spatial extent thresholds; and (4) were published in English with peer review. In studies that met the aforementioned inclusion criteria, the largest group size was selected if the data overlapped with the intersubgroups or with another study. The studies were excluded if they suffered from at least one of the following deficiencies: (1) sufficient data could not be obtained even when more information was asked from the corresponding authors by phone or email; (2) there were fewer than nine subjects in either AD group or HC group; (3) the data overlapped with those of another publication; (4) there were uncorrected results and the spatial extent threshold was not reported; and (5) there was no $\mathrm{HC}$ group; (6) studies limit their analyses to specific ROI; (7) the patient-group included subgroups of "vascular" AD. and (8) it was not clear if the coordinates were in the Talairach or MNI (Montreal Neurological Institute) space (necessary for the text files in SDM software). The method used in the current study was in accordance with the Meta-analysis Of Observational Studies in Epidemiology (MOOSE) guidelines for meta-analyses of observational studies [10].

\section{Data extraction}

The coordinates in each study were independently extracted by two neurologists (Wang WY and Yin RH) according to the ES-SDM method [9].

\section{Voxel-based meta-analysis (VBM)}

The ROI is one of the most commonly used methods to address morphometric changes in the brain [2]. This method manually drew and calculated the brain regions of interest by investigators, then compared their volume in $\mathrm{AD}$ to HC. However, major limitations of the ROI approaches are that it requires a priori decision concerning which structures need to be evaluated and that regions showing abnormal GM volume might be part of a large ROI, or spread over different ROIs, thereby potentially reducing statistical power of the underlying morphological analysis [4]. This analysis was performed in a standard process using the SDM software (http://www. sdmproject.com/-software/) to compare the GM changes between the $\mathrm{AD}$ and $\mathrm{HC}$ groups. A systematic wholebrain voxel-based jackknife sensitivity analysis was performed to test the replicability of the results. All these processes were referred to the SDM Tutorial (http:// sdmproject.com/software/Tutorial.pdf) and publications. The full-width at half maximum (FWHM) in SDM are set at $20 \mathrm{~mm}$ because in previous simulations it has been found to have an excellent control of false positives results in a preprocessing step [9]. The statistical threshold was set to be a p-value of $<0.005$ without correction for false discovery rate (FDR) as this was found to optimally balance sensitivity and specificity [9]. Residual heterogeneity was not significant $(\tau=0.154, Q=83.926, d f=28$, $\mathrm{P}=1.79 \times 10^{-7}$ ). The SDM software editor was also contacted by email when necessary.

\section{Result}

\section{Included studies and sample characteristics}

The initial literature search identified 821 potentially relevant articles, of which 68 met the inclusion criteria. After full text screening, 38 articles were excluded for different reasons (Figure 1). Finally, 30 [7,11-39] articles published between 1995 and 2014 met the selection criteria and had accessible information concerning grey matter changes between $\mathrm{AD}$ and $\mathrm{HC}$. The clinical and demographic data of participants in all included studies 


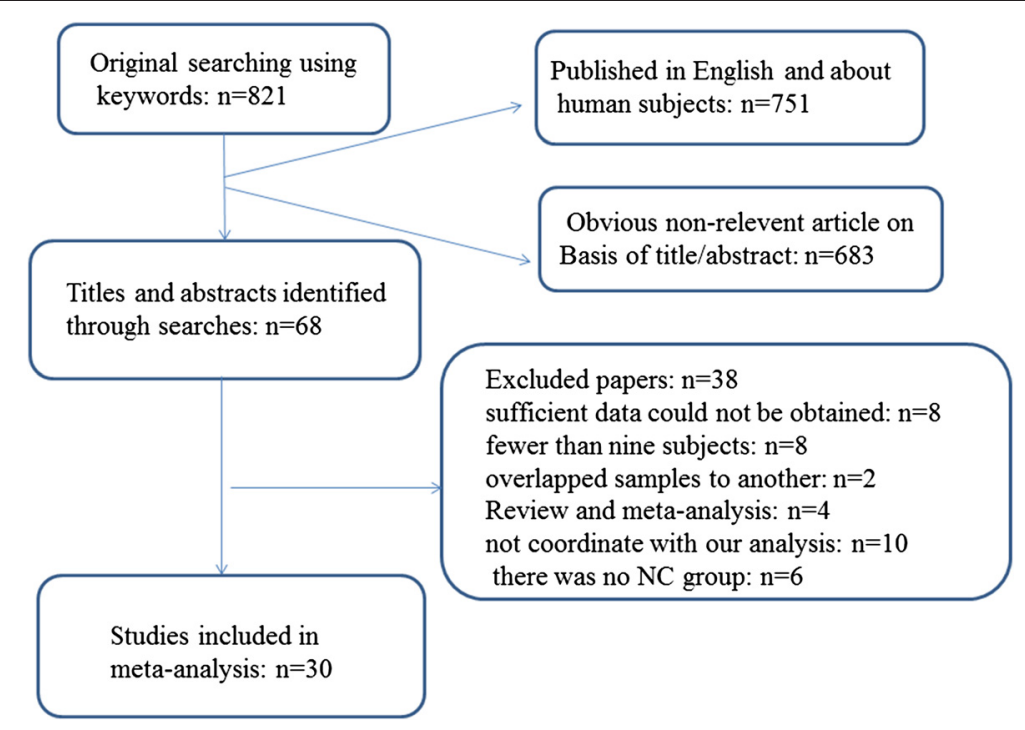

Figure 1 Flowchart describing the approach used to identify all eligible studies of meta-analysis.

are presented in Table 1 . The technical details of the included studies are shown in Table 2. A total of 960 people with $\mathrm{AD}$ and $1195 \mathrm{HC}$ were included. In each study, no statistically significant difference was found in age, gender between the $\mathrm{AD}$ and $\mathrm{HC}$, as the original studies were already well matched in this respect. Sensitivity analysis was first used and no outliers were found in this study.

\section{Global GM volumes}

This analysis was not carried out because of the small number of studies with a detailed global GM density or volume.

\section{Regional differences}

The included studies reported GM reductions at 334 coordinates in AD compared with NC. A group comparison of $\mathrm{AD}$ patients and $\mathrm{HC}$ was carried out. AD patients had considerable smaller GMV in the limbic regions (left parahippocampl gyrus and left posterior cingulate gyrus). In addition, there were GM decreases in right fusiform gyrus and right superior frontal gyrus (as shown in Table 3 and Figure 2) in patients with AD from the SDM map threshold of $\mathrm{P}<0.005$ with voxels $>10$. The patients with AD had no significant GM increase in any region compared with the $\mathrm{HC}$ subjects in all included studies.

\section{Sensitivity analysis}

A whole-brain voxel-based jackknife sensitivity analysis was conducted to test the replicability of the results. This consists of repeating the main statistical analysis 30 times but systematically removing one different study each time to ensure that no single study will bias the combined results and recalculating the stability of the remaining studies. As shown in Table 3, the whole brain jackknife sensitivity analysis indicated a GM reduction in left parahippocampal gyrus and left posterior cingulate gyrus highly replicable because they were preserved throughout all of the 30 combinations of studies. Grey matter decreases in right fusiform gyrus failed to emerge in one of the study and right superior frontal gyrus failed to emerge in two of the study.

\section{Analyses of subgroups}

The above results were highly reproducible when the analyses were repeated and limited to $12-\mathrm{mm}$ smoothing kernel or $1.5 \mathrm{~T}$ MRI scanner to remove the potential confounding effects of methodological differences.

\section{Meta-regression}

The meta-regression analysis showed that the higher neuropsychological test scores in AD (Mini Mental State Examination (MMSE) scores- used as a concise screening tool that assesses the severity of cognitive impairment and reflects cognitive in the progression of $\mathrm{AD}$ [40], available in all the studies) was associated with decreased grey matter volumes in the left parahippocampal gyrus $(-18,-16,-28$; SDM-Z $=-3.963$; voxels $=490$; $\left.\mathrm{P}=3.0965 \times 10^{-5}\right)$.

\section{Discussion}

To our knowledge, this is the first meta-analysis of voxel-based morphometry studies of grey matter volume in $\mathrm{AD}$ and $\mathrm{HC}$ subjects using ES-SDM software. The present voxel-wise meta-analysis mainly found that 
Table 1 Demographic and clinical characteristics of VBM studies for GMV on AD in meta-analysis

\begin{tabular}{|c|c|c|c|c|c|c|c|c|c|}
\hline \multirow[t]{2}{*}{ Study } & \multicolumn{2}{|l|}{$\begin{array}{l}\text { Number } \\
\text { (Female) }\end{array}$} & \multicolumn{2}{|l|}{$\begin{array}{l}\text { Age } \\
\text { (Years) }\end{array}$} & \multicolumn{2}{|l|}{$\begin{array}{l}\text { Education } \\
\text { (Years) }\end{array}$} & \multicolumn{2}{|l|}{ MMSE } & \multirow[t]{2}{*}{ Diagnostic criteria } \\
\hline & $\overline{A D}$ & NC & $\overline{A D}$ & NC & $\overline{A D}$ & NC & $A D$ & NC & \\
\hline Guo2014 [11] & $35(23)$ & $27(11)$ & $72.4(8.5)$ & $69.2(6.5)$ & NA & NA & $19.7(4.1)$ & $28.9(1.0)$ & ICD-10 criteria \\
\hline Teipel2012 [30] & $137(79)$ & $143(72)$ & $72.5(8.3)$ & $69.2(5.9)$ & $10.2(3.3)$ & $13.1(3.8)$ & $20.6(5.3)$ & $28.8(1.1)$ & NINCDS-ADRDA \\
\hline Rami2012 [12,62] & 32 & 24 & $75.5(5.5)$ & $71.4(6.6)$ & $8.3(2.9)$ & $9.0(4.7)$ & $22.5(3.3)$ & $28.1(1.4)$ & NINCDS-ADRDA \\
\hline Gili2011 [29] & $11(4)$ & $10(3)$ & $71.9(7.9)$ & $64.1(10.5)$ & $9.9(4.9)$ & $14.3(3.4)$ & $19.7(4.5)$ & $28.34(2.0)$ & NINCDS-ADRDA \\
\hline Lehmann2011 [13] & $30(16)$ & $50(33)$ & $69.2(8.9)$ & $63.7(9.6)$ & NA & NA & NA & NA & NINCDS-ADRDA \\
\hline Serra2010 [14] & $9(6)$ & $13(4)$ & $72.4(7.5)$ & $64.1(10.5)$ & $9.9(4.9)$ & $14.3(3.4)$ & $18.2(4.4)$ & $28.9(1.3)$ & NINCDS-ADRDA \\
\hline Guo2010 [23] & $13(7)$ & $14(8)$ & $72.1(6.5)$ & $70.4(3.5)$ & NA & NA & $18.5(3.5)$ & $28.5(0.6)$ & NINCDS-ADRDA \\
\hline Ibrahim2009 [22] & $20(11)$ & $23(18)$ & $73.67(7.58)$ & $66.70(5.82)$ & $13.10(2.95)$ & $14.22(2.36)$ & $15.60(7.20)$ & $29.13(0.99)$ & NINCDS-ADRDA \\
\hline Kanda2008 [15] & 20 & 20 & 65.0 & 65.2 & NA & NA & 17.5 & 29.0 & NINCDS-ADRDA \\
\hline Whitwell2007 [5,16] & $38(22)$ & $38(22)$ & $65.3(6.9)$ & $65.9(7.0)$ & 12.5 & 14.0 & 17.0 & 29.0 & NINCDS-ADRDA \\
\hline Hamalainen2007 [24] & $15(10)$ & $21(17)$ & $73.1(6.7)$ & $71.2(4.9)$ & $8.2(2.7)$ & $7.9(2.9)$ & $21.7(3.7)$ & $27.7(2.0)$ & NINCDS-ADRDA \\
\hline DiPaola2007 [18] & $18(14)$ & $18(14)$ & $64.3(10.2)$ & $65.4(10.6)$ & $8.8(4.2)$ & $8.1(2.8)$ & $16.9(4.3)$ & $29.0(1.6)$ & NINCDS-ADRDA \\
\hline Samuraki2007 [28] & $39(20)$ & $73(36)$ & $68.03(8.76)$ & $66.78(8.34)$ & NA & NA & $22.3(3.3)$ & $29.2(0.8)$ & NINCDS-ADRDA \\
\hline Rabinovici2007 [17] & $11(6)$ & $40(23)$ & $64.5(9.7)$ & $63.5(5.8)$ & $16.5(2.9)$ & $17.4(2.4)$ & $19.9(6.9)$ & $29.7(0.5)$ & NINCDS-ADRDA \\
\hline Bozzali2006 [26] & $22(11)$ & $20(13)$ & $67.9(7.6)$ & $65.8(6.8)$ & NA & NA & $19.8(4.1)$ & $27.3(1.2)$ & NINCDS-ADRDA \\
\hline Shiino2006 [27] & $40(21)$ & $88(48)$ & $71.1(9.7)$ & $68.7(8.7)$ & NA & NA & $18.03(3.91)$ & $29.09(1.47)$ & NINCDS-ADRDA \\
\hline Ishii2005 [19] & $30(22)$ & $30(22)$ & $66.8(7.0)$ & $66.8(7.9)$ & NA & NA & $14.7(5.4)$ & $24.0(2.2)$ & NINCDS-ADRDA \\
\hline Hirata2005 [7] & $61(29)$ & $82(43)$ & $70.6(8.4)$ & $70.1(7.7)$ & NA & NA & $26.0(1.5)$ & $28.7(1.5)$ & NINCDS-ADRDA \\
\hline Boxer2003 [20] & $11(8)$ & $15(7)$ & $69.6(8.2)$ & $65.1(8.3)$ & $16.3(3.8)$ & $16.6(3.9)$ & $20.2(7.3)$ & $29.5(0.5)$ & NINCDS-ADRDA \\
\hline Frisoni2002 [21] & $29(23)$ & $26(17)$ & $74(9)$ & $69(8)$ & $7(4)$ & $8(3)$ & $21(4)$ & $29(1)$ & NINCDS-ADRDA \\
\hline Ohnishi2001 [25] & $26(15)$ & $47(16)$ & $72.1(1.1)$ & $28.5(6.0)$ & NA & NA & $20.7(3.1)$ & NA & NINCDS-ADRDA \\
\hline Matsuda2002 [34] & $15(4)$ & $25(9)$ & $71.1(7.1)$ & $71.2(7.3)$ & NA & NA & $21.5(2.9)$ & $29.5(0.6)$ & DSM-IV \\
\hline Busatto2003 [35] & $14(5)$ & $14(6)$ & $72.2(7.2)$ & $69.4(5.9)$ & $9.1(4.2)$ & $7.1(4.7)$ & $20.7(3.1)$ & $29.1(0.5)$ & NINCDS-ADRDA \\
\hline Raji2009 [31] & $33(20)$ & $169(96)$ & $82.8(5.16)$ & $77.57(3.62)$ & NA & NA & NA & NA & NINCDS-ADRDA \\
\hline Honea2009 [32] & $61(37)$ & $56(33)$ & $74.3(6.3)$ & $73.3(6.2)$ & $15.3(3.3)$ & $16.4(2.2)$ & $26.2(3.7)$ & $29.4(0.8)$ & NINCDS-ADRDA \\
\hline Brenneis2004 [36] & $10(3)$ & $10(6)$ & $73.1(7.6)$ & $65.1(8.1)$ & NA & NA & $17.4(7.9)$ & $28.8(1.6)$ & DSM-IV \\
\hline Hirao2006 [39] & $61(29)$ & $61(31)$ & $70.6(8.4)$ & $70.2(7.3)$ & NA & NA & $26.0(1.5)$ & $28.7(1.5)$ & NINCDS-ADRDA \\
\hline Brambati2009 [33] & $10(5)$ & $13(8)$ & $71.5(5.9)$ & $75.0(5.0)$ & $12.6(4.7)$ & $14.9(5.0)$ & $22.5(2.3)$ & $29.1(1.2)$ & NINCDS-ADRDA \\
\hline Baxter2006 [38] & $15(4)$ & $15(8)$ & $75.5(7.8)$ & $76.4(7.9)$ & $14.7(2.9)$ & $15.3(2.9)$ & $14-28$ & $28.5(1.1)$ & NINCDS-ADRDA \\
\hline Zahn2005 [37] & $10(6)$ & $10(5)$ & $66.5(8.9)$ & $65.8(7.8)$ & NA & NA & $23.6(2.8)$ & NA & NINCDS-ADRDA \\
\hline
\end{tabular}

Key: AD, Alzheimer's disease; NC, normal cognition subjects; MMSE, the Mini Mental State Examination; NA, not available; VBM, voxel-based morphometry; GMV, Grey matter volume; NINCDS-ADRDA, National Institute of Neurological and Communicative Disorders and Stroke/Alzheimer's Disease and Related Disorders Association; DSM, Diagnostic and Statistical Manual of Mental Disorders; ICD-10 criteria, International Classification of Diseases, 10th Revision.

patients with AD have regional GM volume reductions in the limbic regions (left parahippocampl gyrus and left posterior cingulate gyrus). In addition, there were GM decreases in right fusiform gyrus and right superior frontal gyrus, medial orbital. The results remained largely unchanged when jackknife sensitivity analysis was performed. This indicates that the results were robust and highly replicable.

It has been widely accepted that at rest state, important brain areas-posterior cingulate cortex combines precuneus, lateral temporal cortex, medial prefrontal cortex, and inferior parietal lobule organized into a functionally relevant networks, the "default mode network" (DMN) [41], which is correlated with episodic memory functioning. Several studies have demonstrated that AD is associated with DMN resting state functional MRI disruptions compared to $\mathrm{HC}$, which is marked by abnormalities in structural interactions and functional connectivity [42-44]. In a previous study using single photon emission computed tomography (SPECT), Pagani et al. proposed that posterior cingulate cortex covaried with the left lateral parietal lobe [45]. In addition, Jacobs et al. demonstrated 
Table 2 Technique details of VBM studies for GMV on AD in meta-analysis

\begin{tabular}{|c|c|c|c|c|c|}
\hline Study & Scanner $(T)$ & Software & FHWH $(\mathrm{mm})$ & P-value & Coordinates \\
\hline Guo2014 [11] & 3 & SPM8 & 6 & $P<0.05$ (FDR-corrected) & 14 \\
\hline Teipel2012 [30] & $1.5(3)$ & SPM8 & 8 & $P<0.001$, uncorrected for multiple comparisons & 22 \\
\hline Rami2012 [12,62] & 3 & SPM5 & 8 & $P<0.0001$ (uncorrected) & 4 \\
\hline Gili2011 [29] & 3 & SPM5 & 12 & $P<0.001$ uncorrected & 9 \\
\hline Lehmann2011 [13] & 1.5 & SPM5 & 6 & Multiple-comparison correction FDR $<0.05$ & 1 \\
\hline Serra2010 [14] & 3 & SPM5 & 12 & $P<0.05$ (FEW corrected) & 17 \\
\hline Guo2010 [23] & 3 & SPM5 & 8 & $P<0.05$ (FDR Corrected) & 16 \\
\hline Ibrahim2009 [22] & 1.5 & SPM5 & 10 & $\mathrm{p}$-value $<0.005$ with the FDR corrected & 15 \\
\hline Kanda2008 [15] & 1.5 & SPM2 & 12 & $P<0.01$, corrected & 7 \\
\hline Whitwell2007 [5,16] & 1.5 & SPM5 & 8 & $P<0.05$, corrected for multiple comparisons & 1 \\
\hline Hamalainen2007 [24] & 1.5 & SPM5 & 12 & $P<0.05$, corrected & 19 \\
\hline DiPaola2007 [18] & 1.5 & SPM5 & 10 & $P<0.05$ (FWE corrected) & 18 \\
\hline Samuraki2007 [28] & 1.5 & SPM2 & 12 & $P<0.001$, correct for multiple comparisons & 5 \\
\hline Rabinovici2007 [17] & 1.5 & SPM2 & 12 & $\mathrm{P}<0.05$ (FWE- corrected) & 19 \\
\hline Bozzali2006 [26] & 1.5 & SPM2 & 12 & $\mathrm{P}<0.05$ corrected & 19 \\
\hline Shiino2006 [27] & 1.5 & SPM99 & 12 & $P<0.05$ corrected & 16 \\
\hline Ishii2005 [19] & 1.5 & SPM5 & 12 & $P<0.05$, corrected & 3 \\
\hline Hirata2005 [7] & 1 & SPM5 & 12 & $P<0.001$, correction for multiple non-independent comparisons & 2 \\
\hline Boxer2003 [20] & 1.5 & SPM5 & 12 & $P<0.05$, corrected for multiple comparisons & 3 \\
\hline Frisoni2002 [21] & 1.5 & SPM99 & 8 & $P<0.05$, corrected for multiple comparisons & 34 \\
\hline Ohnishi2001 [25] & 1.0 & SPM96 & 12 & $P<0.001$, correct for multiple comparisons & 2 \\
\hline Matsuda2002 [34] & 1.0 & SPM99 & 12 & $\mathrm{P}<0.05$ correct for multiple comparisons & 13 \\
\hline Busatto2003 [35] & 1.5 & SPM99 & 8 & $P<0.001$, uncorrected & 9 \\
\hline Raji2009 [31] & 1.5 & SPM2 & 10 & $P<0.05$ (FDR Corrected) & 5 \\
\hline Honea2009 [32] & 3.0 & SPM5 & 10 & $P<0.05$, FWE corrected & 13 \\
\hline Brenneis2004 [36] & 1.5 & SPM99 & 8 and 10 & $\mathrm{P}<0.05$ corrected for small volumes & 14 \\
\hline Hirao2006 [39] & 1.0 & SPM2 & 12 & $P<0.001$; corrected for multiple comparisons & 2 \\
\hline Brambati2009 [33] & 3.0 & SPM5 & 8 & $\mathrm{p}<0.001$ uncorrected & 17 \\
\hline Baxter2006 [38] & 1.5 & SPM2 & 12 and 8 & p-values $<0.0001$ uncorrected & 6 \\
\hline Zahn2005 [37] & 1.5 & SPM2 & 8 & $P<0.001$ uncorrected & 4 \\
\hline
\end{tabular}

Key: AD, Alzheimer's disease; FDR, false discovery rate; FEW, family-wise error; FWHM, full width at half-maximum; SPM, Statistical Parametric Mapping; T, Tesla; VBM, voxel-based morphometry; GMV, grey matter volume.

that grey matter atrophy in inferior parietal lobule was connected to the prefrontal cortices [46]. Recently, Wang and colleagues employing Bayesian network models and integrating grey matter volume information from multiple brain regions found increased correlations from Left inferior temporal cortex to Left hippocampus, Left hippocampus to Right inferior temporal cortex, Right hippocampus to Right inferior temporal cortex, and Right inferior parietal cortex to Posterior cingulate cortex in $\mathrm{AD}$ patients [47]. The morphological changes in the grey matter in different brain regions abide by covariance pattern, reflecting the DMN network attributes of the human brain, and also suggest that the atrophy of these structures is not independent, but that primary neurodegeneration in one of the structures that could lead to secondary degeneration of regions connected to it.

A previous reported meta-analysis of ALE structural MRI studies found evidence of volume reductions in the medial temporal lobes (MTL) (including entorhinal cortex, hippocampus, parahippocampus, amygdala and uncus), temporal, frontal and cingulate cortices [48]. This is in line with our meta-analysis. However, our study did not found volume reductions in parietal and insular cortices. This may be due to the inclusion of recently available data and the improvement of meta-analytic method.

Grey matter differed in AD and Dementia with Lewy bodies (DLB) when compared the result of the 
Table 3 Regional differences in grey matter volume between individuals with AD and HC

\begin{tabular}{|c|c|c|c|c|c|c|}
\hline \multirow[t]{2}{*}{ Region } & \multicolumn{4}{|l|}{ Maximum } & \multirow{2}{*}{$\begin{array}{l}\text { Cluster } \\
\text { Clusters breakdown } \\
\text { (no. of voxels) }\end{array}$} & \multirow{2}{*}{$\begin{array}{l}\text { Jackknife sensitivity analysis } \\
\text { (combination of studies } \\
\text { detecting the differences) }\end{array}$} \\
\hline & $\begin{array}{l}\text { MNI coordinates } \\
x y z\end{array}$ & SDM value & $P$ value & $\begin{array}{l}\text { Number } \\
\text { of voxels }\end{array}$ & & \\
\hline \multirow{6}{*}{$\begin{array}{l}\text { Left parahippocampl } \\
\text { gyrus, BA36 }\end{array}$} & \multirow[t]{6}{*}{$-30,-10,-28$} & \multirow[t]{6}{*}{-6.544} & \multirow[t]{6}{*}{$\sim 0$} & \multirow[t]{6}{*}{3440} & Left parahippocampal gyrus (676) & \multirow[t]{6}{*}{30 out of 30} \\
\hline & & & & & Left insula (515) & \\
\hline & & & & & Left temporal gyrus (879) & \\
\hline & & & & & Left hippocampus (706) & \\
\hline & & & & & Left inferior frontal gyrus (450) & \\
\hline & & & & & Left rolandic operculum (214) & \\
\hline \multirow[t]{5}{*}{ Right fusiform gyrus } & \multirow[t]{5}{*}{$34,-8,-30$} & \multirow[t]{5}{*}{-6.257} & \multirow[t]{5}{*}{$\sim 0$} & \multirow[t]{5}{*}{5838} & Right temporal gyrus (3300) & \multirow[t]{5}{*}{29 out of 30} \\
\hline & & & & & Right parahippocampal gyrus (813) & \\
\hline & & & & & Right hippocampus (521) & \\
\hline & & & & & Right rolandic operculum (447) & \\
\hline & & & & & Right insula (757) & \\
\hline \multirow{3}{*}{$\begin{array}{l}\text { Left posterior cingulate } \\
\text { gyrus, BA } 23\end{array}$} & \multirow[t]{3}{*}{$-8,-48,32$} & \multirow[t]{3}{*}{-3.880} & \multirow[t]{3}{*}{$\sim 0$} & \multirow[t]{3}{*}{1866} & Left /Right precuneus (1056) & \multirow[t]{3}{*}{30 out of 30} \\
\hline & & & & & $\begin{array}{l}\text { Left/Right posterior cingulate } \\
\text { gyrus (358) }\end{array}$ & \\
\hline & & & & & $\begin{array}{l}\text { Left/Right median cingulate/ } \\
\text { paracingulate gyri (452) }\end{array}$ & \\
\hline \multirow{3}{*}{$\begin{array}{l}\text { Right superior frontal } \\
\text { gyrus, medial orbital, } \\
\text { BA } 11\end{array}$} & \multirow[t]{3}{*}{$4,34,-12$} & \multirow[t]{3}{*}{-3.362} & \multirow[t]{3}{*}{0.000474795} & \multirow[t]{3}{*}{221} & $\begin{array}{l}\text { Left/Right superior frontal gyrus, } \\
\text { medial orbital (84) }\end{array}$ & \multirow[t]{3}{*}{28 out of 30} \\
\hline & & & & & Left /Right gyrus rectus (23) & \\
\hline & & & & & $\begin{array}{l}\text { Left anterior cingulate/ } \\
\text { paracingulate gyri (18) }\end{array}$ & \\
\hline
\end{tabular}

Regions identified by meta-analysis of coordinates from Twenty one studies (voxelwise $\mathrm{p}<0.005$ and FWHM $20 \mathrm{~mm}$ ).

Key: AD, Alzheimer's disease; HC, healthy controls; MNI, Montreal Neurological Institute; SDM, signed differential mapping; GMV, Grey matter volume; BA, Brodmann area.

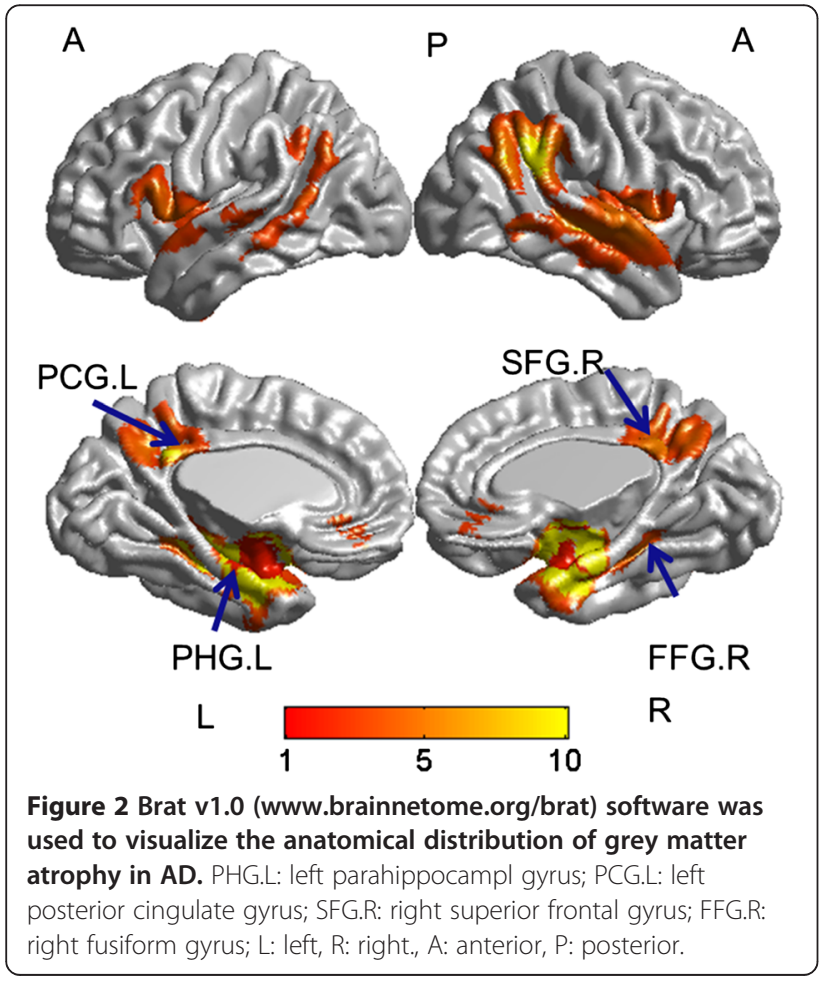

meta-analysis using the SDM methodologies. AD was characterized by GMV decreased in the MTL but not the lateral temporal lobe are coincident with the recently published meta-analysis showed that medial temporal lobe structures were relative preserved in DLB compared with $\mathrm{AD}$ [49]. In AD, the MTL have been proved associated with the degree of memory, regions which are involved in encoding and retrieval of episodic and spatial memory [50,51]. Pathological investigations in AD confirmed our findings, where the neurofibrillary tangles and amyloid plaques observed from the beginning [52], and subsequently affects the posterior limbic system due to its close connections to the posterior sector of the cingulate gyrus. So far we could not find the Tau pathology are connected with GMV loss in AD in MTL. Furthermore, the postmortem autopsy also have been testified it as cardinal structures affected with loss of neurons in patients with AD [53] of which may explanation of the MTL atrophy and supports our results. A previous meta-analysis of structural and functional imaging studies also revealed consistent volumetric reduction within the MTL was the most sensitive measure to identify AD in patients with a duration of illness greater than 4 years [54]. The volumetric reductions seen in this region may 
help explain reports of episodic memory loss in early stages of AD.

One of the key findings of the present study is the GM volume reduction in the left parahippocampal gyrus, the most important cortical input and output region of the hippocampus and mediates corticohippocampal communication. An important study by Burgmans et al. found the association with memory decline is larger in the posterior parahippocampus than in the hippocampus and entorhinal cortex [55], and parahippocampal gyrus atrophy in AD are related to patients' anterograde memory impairments. In addition, the finding of robust GM loss in the parahippocampal gyrus is in line with one previous ROIbased quantitative volumetric MRI study [56]. Previous histological studies have already revealed that entorhinal cortex as the earliest neuropathological changes in AD patients, which is the anterior part of the parahippocampal gyrus [57]. Abnormalities in this region is also confirmed by previous researches through other neuroimaging methods such as functional MRI (fMRI) [58], technetium (Tc-99 m) hexamethylpropyleneamine oxime (TC-99 m HMPAO) SPECT [59] and Pittsburgh Compound B [60].

Our meta-analysis revealed grey matter reductions in left posterior cingulate gyrus. Evidence from the positron emission tomography (PET) studies using [11C]PiB (Pittsburgh compound B) binds to amyloid, have identified that this region was related to the disease process [61]. The finding of robust GM loss in parietal regions is in line with a recent fMRI study, which have found atrophy in this region in the very early progression of $\mathrm{AD}$ [62]. This result is also in accordance with previous Fluorodeoxyglucose (FDG) PET studies which reported metabolic decline in the posterior cingulate cortex of patients with AD [63]. In addition, using dynamic susceptibility contrast magnetic resonance imaging, Hauser and colleagues have detected that the posterior cingulate gyrus perfusion was significantly decreased in patients with AD compared to patients with MCI or HC [64]. Likewise, Yoshida et al. revealed decreased regional cerebral blood flow and regional cerebral protein synthesis in this region. The probable explanation of the posterior cingulate gyrus atrophy is that neuronal atrophy and the fibrillary amyloid deposition which need further investigation.

The current study has a number of strengths. The most importantly utilized SDM methodologies, a wellvalidated, automated method of meta-analyzing data from multiple VBM studies using the reported peak coordinates to recreate (to a limited extend) the original maps, thus accounting for both positive and negative differences [9]. This technique has already been successfully applied in a number of previous meta-analysis of VBM studies on several neurologic and neuropsychological disorders such as amyotrophic lateral sclerosis
[65], obsessive-compulsive disorder [66], bipolar disorder [67] and DLB [49]. The ES-SDM that we used in this study is a new version of the SDM meta-analytic method featuring two methodological improvements: combining peak coordinates and statistical parametric maps and use of well-established statistics accounting for within- and between-study variance [9]. The new version-ES-SDM has been proved to be valid and superior to previous coordinate-based meta-analytical methods such as ALE and the default settings, and also optimizes the sensitivity while protecting against the false positives [9].

\section{Limitations}

There are several methodological limitations of this study, some of which are inherent to all meta-analytical approaches. One limitation is the accuracy of the results, because peak-based meta-analyses are based on pooling of stereotactic coordinates rather than on raw statistical brain maps, and this may lead to less accurate results. Nevertheless, obtaining and analysing the raw images from these studies is logistically and technically difficult. Second, methodological differences of VBM studies, for instance different preprocessing protocols (traditional or optimized), smoothing kernels, and statistical thresholding methods cannot be entirely ruled out even if a subgroup analysis was performed. Third, as mentioned above, our regression analyses should be taken cautiously because they included a small number of studies and variability in the data was limited. Fourth, several of the included studies reported grey matter density rather than volume. The mean density of the GM is derived from the percentage of absolute GM volume divided by total brain volume, which might result in different locations of deficit areas from results achieved by VBM measurements of GM volume. The meta-analysis will subsequently influence the results by different locations. Finally, although voxel-wise metaanalytical methods provide excellent control for falsepositive results, it is difficulty to avoiding false-negative results completely. Since the SDM approach does not use effect sizes for non-significant changes (e.g., those that have $\mathrm{p}<0.005$ and do not survive correction for multiple comparisons), in some regions with some trends towards significant or even just differences that appear not to be significant because of the effect size, it might cause false negative findings.

\section{Conclusion}

The results of meta-analysis implicate regional grey matter reduction in $\mathrm{AD}$ is a functionally relevant networks (DMN) of prefrontal, limbic, temporal regions involved in the episodic memory functioning and attentional processing compared with $\mathrm{HC}$. A better understanding of the neural network implicated in $\mathrm{AD}$ may inform the diagnosis and treatment of this condition in the future. 


\section{Competing interests}

The authors declare that they have no competing interests.

\section{Authors' contributions}

JTY and Lan Tan design the whole study. YL revised the article and prepared one figure. WYW searched and selected the studies, analyzed the data, prepared figures and drafted the article. RHY, HFW, JW, and Lin Tan participated in the acquisition of data and statistical analysis. WYW and RHY participated in the interpretation of data. All authors read and approved the final manuscript.

\section{Acknowledgments}

We thank all the authors of the included studies especially Dr Radua who helped us with the ES-SDM software problems. This work was supported by grants from the National Natural Science Foundation of China (81471309, 81371406, 81171209), the Natural Science Foundation of Beijing (No.7152096), the Shandong Provincial Outstanding Medical Academic Professional Program, Shandong Provincial Collaborative Innovation Center for Neurodegenerative Disorders, Qingdao Key Health Discipline Development Fund, and Qingdao Outstanding Health Professional Development Fund.

\section{Author details}

${ }^{1}$ Department of Neurology, Qingdao Municipal Hospital, School of Medicine, Qingdao University, No.5 Donghai Middle Road, Qingdao, Shandong Province 266071, China. ${ }^{2}$ College of Medicine and Pharmaceutics, Ocean University of China, Qingdao 266011, China. '3epartment of Neurology, Qingdao Municipal Hospital, Nanjing Medical University, Nanjing 266071, China. ${ }^{4}$ Brainnetome Center, Institute of Automation, Chinese Academy of Sciences, Beijing 100190, China. ${ }^{5}$ National Laboratory of Pattern Recognition, Institute of Automation, Chinese Academy of Sciences, Beijing 100190, China. ${ }^{6}$ Department of Psychosis Studies, Institute of Psychiatry, King's College London, London, UK. ${ }^{7}$ Research Unit, FIDMAG Germanes Hospitala'riesCIBERSAM, Sant Boi de Llobregat, Barcelona, Spain.

\section{Received: 8 November 2014 Accepted: 18 March 2015}

\section{Published online: 27 March 2015}

\section{References}

1. Pereira JM, Xiong L, Acosta-Cabronero J, Pengas G, Williams GB, Nestor PJ. Registration accuracy for VBM studies varies according to region and degenerative disease grouping. Neurolmage. 2010;49(3):2205-15. doi:10.1016/j.neuroimage.2009.10.068.

2. Busatto GF, Diniz BS, Zanetti MV. Voxel-based morphometry in Alzheimer's disease. Expert Rev Neurother. 2008;8(11):1691-702. doi:10.1586/ 14737175.8.11.1691.

3. Costafreda SG, David AS, Brammer MJ. A parametric approach to voxel-based meta-analysis. Neurolmage. 2009;46(1):115-22. doi:10.1016/j.neuroimage.2009.01.031.

4. Ferreira LK, Diniz BS, Forlenza OV, Busatto GF, Zanetti MV. Neurostructural predictors of Alzheimer's disease: a meta-analysis of VBM studies. Neurobiol Aging. 2011;32(10):1733-41. doi:10.1016/j.neurobiolaging.2009.11.008.

5. Whitwell JL, Przybelski SA, Weigand SD, Knopman DS, Boeve BF, Petersen RC, et al. 3D maps from multiple MRI illustrate changing atrophy patterns as subjects progress from mild cognitive impairment to Alzheimer's disease. Brain. 2007;130(Pt 7):1777-86. doi:10.1093/brain/awm112.

6. Karas GB, Burton EJ, Rombouts SA, van Schijndel RA, O'Brien JT, Scheltens P, et al. A comprehensive study of gray matter loss in patients with Alzheimer's disease using optimized voxel-based morphometry. NeuroImage. 2003;18(4):895-907.

7. Hirata Y, Matsuda H, Nemoto K, Ohnishi T, Hirao K, Yamashita F, et al. Voxel-based morphometry to discriminate early Alzheimer's disease from controls. Neurosci Lett. 2005;382(3):269-74. doi:10.1016/j.neulet.2005.03.038.

8. Whitwell JL, Jack Jr CR, Przybelski SA, Parisi JE, Senjem ML, Boeve BF, et al. Temporoparietal atrophy: a marker of AD pathology independent of clinical diagnosis. Neurobiol Aging. 2011;32(9):1531-41. doi:10.1016/j. neurobiolaging.2009.10.012.

9. Radua J, Mataix-Cols D, Phillips ML, El-Hage W, Kronhaus DM, Cardoner N, et al. A new meta-analytic method for neuroimaging studies that combines reported peak coordinates and statistical parametric maps. Eur Psychiatry. 2012;27(8):605-11. doi:10.1016/j.eurpsy.2011.04.001.
10. Stroup DF, Berlin JA, Morton SC, Olkin I, Williamson GD, Rennie D, et al. Meta-analysis of observational studies in epidemiology: a proposal for reporting. Meta-analysis Of Observational Studies in Epidemiology (MOOSE) group. JAMA. 2000;283(15):2008-12.

11. Guo Y, Zhang Z, Zhou B, Wang P, Yao H, Yuan M, et al. Grey-matter volume as a potential feature for the classification of Alzheimer's disease and mild cognitive impairment: an exploratory study. Neurosci Bull. 2014;30(3):477-89. doi:10.1007/s12264-013-1432-x.

12. Rami L, Sole-Padulles C, Fortea J, Bosch B, Llado A, Antonell A, et al. Applying the new research diagnostic criteria: MRI findings and neuropsychological correlations of prodromal AD. Int J Geriatr Psychiatry. 2012;27(2):127-34. doi:10.1002/gps.2696.

13. Lehmann M, Crutch SJ, Ridgway GR, Ridha BH, Barnes J, Warrington EK, et al. Cortical thickness and voxel-based morphometry in posterior cortical atrophy and typical Alzheimer's disease. Neurobiol Aging. 2011;32(8):1466-76. doi:10.1016/j.neurobiolaging.2009.08.017.

14. Serra L, Cercignani M, Lenzi D, Perri R, Fadda L, Caltagirone C, et al. Grey and white matter changes at different stages of Alzheimer's disease. J Alzheimers Dis. 2010;19(1):147-59. doi:10.3233/JAD-2010-1223.

15. Kanda T, Ishii K, Uemura T, Miyamoto N, Yoshikawa T, Kono AK, et al. Comparison of grey matter and metabolic reductions in frontotemporal dementia using FDG-PET and voxel-based morphometric MR studies. Eur J Nucl Med Mol Imaging. 2008;35(12):2227-34. doi:10.1007/s00259-008-0871-5.

16. Whitwell JL, Jack Jr CR, Kantarci K, Weigand SD, Boeve BF, Knopman DS, et al. Imaging correlates of posterior cortical atrophy. Neurobiol Aging. 2007;28(7):1051-61. doi:10.1016/j.neurobiolaging.2006.05.026.

17. Rabinovici GD, Seeley WW, Kim EJ, Gorno-Tempini ML, Rascovsky K, Pagliaro TA, et al. Distinct MRI atrophy patterns in autopsy-proven Alzheimer's disease and frontotemporal lobar degeneration. Am J Alzheimers Dis Other Demen. 2007;22(6):474-88. doi:10.1177/1533317507308779.

18. Di Paola M, Macaluso E, Carlesimo GA, Tomaiuolo F, Worsley KJ, Fadda L, et al. Episodic memory impairment in patients with Alzheimer's disease is correlated with entorhinal cortex atrophy. A voxel-based morphometry study. J Neurol. 2007;254(6):774-81. doi:10.1007/s00415-006-0435-1.

19. Ishii K, Kawachi T, Sasaki H, Kono AK, Fukuda T, Kojima Y, et al. Voxel-based morphometric comparison between early- and late-onset mild Alzheimer's disease and assessment of diagnostic performance of z score images. AJNR Am J Neuroradiol. 2005;26(2):333-40.

20. Boxer AL, Rankin KP, Miller BL, Schuff N, Weiner M, Gorno-Tempini ML, et al. Cinguloparietal atrophy distinguishes Alzheimer disease from semantic dementia. Arch Neurol. 2003;60(7):949-56. doi:10.1001/archneur.60.7.949.

21. Frisoni GB, Testa C, Zorzan A, Sabattoli F, Beltramello A, Soininen H, et al. Detection of grey matter loss in mild Alzheimer's disease with voxel based morphometry. J Neurol Neurosurg Psychiatry. 2002;73(6):657-64.

22. Ibrahim I, Horacek J, Bartos A, Hajek M, Ripova D, Brunovsky M, et al. Combination of voxel based morphometry and diffusion tensor imaging in patients with Alzheimer's disease. Neuro Endocrinol Lett. 2009;30(1):39-45.

23. Guo X, Wang Z, Li K, Li Z, Qi Z, Jin Z, et al. Voxel-based assessment of gray and white matter volumes in Alzheimer's disease. Neurosci Lett. 2010;468 (2):146-50. doi:10.1016/j.neulet.2009.10.086.

24. Hamalainen A, Pihlajamaki M, Tanila H, Hanninen T, Niskanen E, Tervo S, et al. Increased fMRI responses during encoding in mild cognitive impairment. Neurobiol Aging. 2007;28(12):1889-903. doi:10.1016/j. neurobiolaging.2006.08.008.

25. Ohnishi T, Matsuda H, Tabira T, Asada T, Uno M. Changes in brain morphology in Alzheimer disease and normal aging: is Alzheimer disease an exaggerated aging process? AJNR Am J Neuroradiol. 2001;22(9):1680-5.

26. Bozzali M, Filippi M, Magnani G, Cercignani M, Franceschi M, Schiatti E, et al. The contribution of voxel-based morphometry in staging patients with mild cognitive impairment. Neurology. 2006;67(3):453-60. doi:10.1212/01. wnl.0000228243.56665.c2.

27. Shiino A, Watanabe T, Maeda K, Kotani E, Akiguchi I, Matsuda M. Four subgroups of Alzheimer's disease based on patterns of atrophy using VBM and a unique pattern for early onset disease. Neurolmage. 2006;33(1):17-26. doi:10.1016/j.neuroimage.2006.06.010.

28. Samuraki M, Matsunari I, Chen WP, Yajima K, Yanase D, Fujikawa A, et al. Partial volume effect-corrected FDG PET and grey matter volume loss in patients with mild Alzheimer's disease. Eur J Nucl Med Mol Imaging. 2007;34(10):1658-69. doi:10.1007/s00259-007-0454-x. 
29. Gili T, Cercignani M, Serra L, Perri R, Giove F, Maraviglia B, et al. Regional brain atrophy and functional disconnection across Alzheimer's disease evolution. J Neurol Neurosurg Psychiatry. 2011;82(1):58-66. doi:10.1136/ jnnp.2009.199935.

30. Teipel SJ, Wegrzyn M, Meindl T, Frisoni G, Bokde AL, Fellgiebel A, et al. Anatomical MRI and DTI in the diagnosis of Alzheimer's disease: a European multicenter study. J Alzheimers Dis. 2012;31 Suppl 3:S33-47. doi:10.3233/ JAD-2012-112118.

31. Raji CA, Lopez OL, Kuller LH, Carmichael OT, Becker JT. Age, Alzheimer disease, and brain structure. Neurology. 2009;73(22):1899-905. doi:10.1212/ WNL.0b013e3181c3f293.

32. Honea RA, Thomas GP, Harsha A, Anderson HS, Donnelly JE, Brooks WM, et al. Cardiorespiratory fitness and preserved medial temporal lobe volume in Alzheimer disease. Alzheimer Dis Assoc Disord. 2009;23(3):188-97. doi:10.1097/WAD.0b013e31819cb8a2.

33. Brambati SM, Belleville S, Kergoat MJ, Chayer C, Gauthier S, Joubert S. Single- and multiple-domain amnestic mild cognitive impairment: two sides of the same coin? Dement Geriatr Cogn Disord. 2009;28(6):541-9. doi:10.1159/000255240

34. Matsuda H, Kitayama N, Ohnishi T, Asada T, Nakano S, Sakamoto S, et al. Longitudinal evaluation of both morphologic and functional changes in the same individuals with Alzheimer's disease. J Nucl Med. 2002;43(3):304-11.

35. Busatto GF, Garrido GE, Almeida OP, Castro CC, Camargo CH, Cid CG, et al. A voxel-based morphometry study of temporal lobe gray matter reductions in Alzheimer's disease. Neurobiol Aging. 2003;24(2):221-31.

36. Brenneis C, Wenning GK, Egger KE, Schocke M, Trieb T, Seppi K, et al. Basal forebrain atrophy is a distinctive pattern in dementia with Lewy bodies. Neuroreport. 2004;15(11):1711-4.

37. Zahn R, Buechert M, Overmans J, Talazko J, Specht K, Ko CW, et al. Mapping of temporal and parietal cortex in progressive nonfluent aphasia and Alzheimer's disease using chemical shift imaging, voxel-based morphometry and positron emission tomography. Psychiatr Res. 2005;140(2):115-31. doi:10.1016/j.pscychresns.2005.08.001.

38. Baxter LC, Sparks DL, Johnson SC, Lenoski B, Lopez JE, Connor DJ, et al. Relationship of cognitive measures and gray and white matter in Alzheimer's disease. J Alzheimers Dis. 2006;9(3):253-60.

39. Hirao K, Ohnishi T, Matsuda H, Nemoto K, Hirata Y, Yamashita F, et al. Functional interactions between entorhinal cortex and posterior cingulate cortex at the very early stage of Alzheimer's disease using brain perfusion single-photon emission computed tomography. Nucl Med Commun. 2006:27(2):151-6.

40. Tombaugh TN, McIntyre NJ. The mini-mental state examination: a comprehensive review. J Am Geriatr Soc. 1992:40(9):922-35.

41. Goto M, Abe O, Aoki S, Hayashi N, Ohtsu H, Takao H, et al. Longitudinal gray-matter volume change in the default-mode network: utility of volume standardized with global gray-matter volume for Alzheimer's disease: a preliminary study. Radiol Phys Technol. 2015;8(1):64-72. doi:10.1007/s12194-014-0295-9.

42. Wu X, Li R, Fleisher AS, Reiman EM, Guan X, Zhang Y, et al. Altered default mode network connectivity in Alzheimer's disease-a resting functional MRI and Bayesian network study. Hum Brain Mapp. 2011;32(11):1868-81. doi:10.1002/hbm.21153.

43. Greicius MD, Srivastava G, Reiss AL, Menon V. Default-mode network activity distinguishes Alzheimer's disease from healthy aging: evidence from functional MRI. Proc Natl Acad Sci U S A. 2004;101(13):4637-42. doi:10.1073/pnas.0308627101.

44. Seeley WW, Crawford RK, Zhou J, Miller BL, Greicius MD. Neurodegenerative diseases target large-scale human brain networks. Neuron. 2009;62(1):42-52. doi:10.1016/j.neuron.2009.03.024.

45. Pagani M, Salmaso D, Rodriguez G, Nardo D, Nobili F. Principal component analysis in mild and moderate Alzheimer's disease-a novel approach to clinical diagnosis. Psychiatr Res. 2009;173(1):8-14. doi:10.1016/j. pscychresns.2008.07.016.

46. Jacobs HI, Van Boxtel MP, Jolles J, Verhey FR, Uylings HB. Parietal cortex matters in Alzheimer's disease: an overview of structural, functional and metabolic findings. Neurosci Biobehav Rev. 2012;36(1):297-309. doi:10.1016/j.neubiorev.2011.06.009.

47. Wang $Y$, Chen $K$, Yao L, Jin Z, Guo X. Structural interactions within the default mode network identified by Bayesian network analysis in Alzheimer's disease. PLoS One. 2013;8(8):e74070. doi:10.1371/journal. pone.0074070.
48. Yang J, Pan P, Song W, Huang R, Li J, Chen K, et al. Voxelwise meta-analysis of gray matter anomalies in Alzheimer's disease and mild cognitive impairment using anatomic likelihood estimation. J Neurol Sci. 2012;316(1-2):21-9. doi:10.1016/j.jns.2012.02.010.

49. Zhong J, Pan P, Dai Z, Shi H. Voxelwise meta-analysis of gray matter abnormalities in dementia with Lewy bodies. Eur J Radiol. 2014;83(10):1870-4. doi:10.1016/j.ejrad.2014.06.014.

50. Schwindt GC, Black SE. Functional imaging studies of episodic memory in Alzheimer's disease: a quantitative meta-analysis. Neurolmage. 2009;45(1):181-90. doi:10.1016/j.neuroimage.2008.11.024.

51. Ranganath C, Ritchey M. Two cortical systems for memory-guided behaviour. Nat Rev Neurosci. 2012;13(10):713-26. doi:10.1038/nrn3338.

52. Smith AD. Imaging the progression of Alzheimer pathology through the brain. Proc Natl Acad Sci U S A. 2002;99(7):4135-7. doi:10.1073/pnas.082107399.

53. Jin K, Peel AL, Mao XO, Xie L, Cottrell BA, Henshall DC, et al. Increased hippocampal neurogenesis in Alzheimer's disease. Proc Natl Acad Sci U S A. 2004;101(1):343-7. doi:10.1073/pnas.2634794100.

54. Zakzanis KK, Graham SJ, Campbell Z. A meta-analysis of structural and functional brain imaging in dementia of the Alzheimer's type: a neuroimaging profile. Neuropsychol Rev. 2003;13(1):1-18.

55. Burgmans $\mathrm{S}$, van Boxtel MP, van den Berg KE, Gronenschild EH, Jacobs $\mathrm{HI}$, Jolles J, et al. The posterior parahippocampal gyrus is preferentially affected in age-related memory decline. Neurobiol Aging. 2011;32(9):1572-8. doi:10.1016/j.neurobiolaging.2009.09.008.

56. Barkhof F, Polvikoski TM, van Straaten EC, Kalaria RN, Sulkava R, Aronen HJ, et al. The significance of medial temporal lobe atrophy: a postmortem MR study in the very old. Neurology. 2007;69(15):1521-7. doi:10.1212/01. wnl.0000277459.83543.99.

57. Echavarri C, Aalten P, Uylings HB, Jacobs HI, Visser PJ, Gronenschild EH, et al. Atrophy in the parahippocampal gyrus as an early biomarker of Alzheimer's disease. Brain Struct Funct. 2011;215(3-4):265-71. doi:10.1007/s00429-010-0283-8.

58. Soldner J, Meindl T, Koch W, Bokde AL, Reiser MF, Moller HJ, et al. Structural and functional neuronal connectivity in Alzheimer's disease: a combined DTI and fMRI study. Nervenarzt. 2012;83(7):878-87. doi:10.1007/s00115-011-3326-3.

59. Park KW, Yoon HJ, Kang DY, Kim BC, Kim S, Kim JW. Regional cerebral blood flow differences in patients with mild cognitive impairment between those who did and did not develop Alzheimer's disease. Psychiatr Res. 2012;203(2-3):201-6. doi:10.1016/j.pscychresns.2011.12.007.

60. Morris JC, Roe CM, Grant EA, Head D, Storandt M, Goate AM, et al. Pittsburgh compound B imaging and prediction of progression from cognitive normality to symptomatic Alzheimer disease. Arch Neurol. 2009:66(12):1469-75. doi:10.1001/archneurol.2009.269.

61. Edison P, Archer HA, Hinz R, Hammers A, Pavese N, Tai YF, et al. Amyloid, hypometabolism, and cognition in Alzheimer disease: an [11C]PIB and [18F]FDG PET study. Neurology. 2007;68(7):501-8. doi:10.1212/01.wnl.0000244749.20056.d4.

62. Rami L, Sala-Llonch R, Sole-Padulles C, Fortea J, Olives J, Llado A, et al. Distinct functional activity of the precuneus and posterior cingulate cortex during encoding in the preclinical stage of Alzheimer's disease. J Alzheimers Dis. 2012;31(3):517-26. doi:10.3233/JAD-2012-120223.

63. Minoshima S, Giordani B, Berent S, Frey KA, Foster NL, Kuhl DE. Metabolic reduction in the posterior cingulate cortex in very early Alzheimer's disease. Ann Neurol. 1997:42(1):85-94. doi:10.1002/ana.410420114.

64. Hauser T, Schonknecht P, Thomann PA, Gerigk L, Schroder J, Henze R, et al. Regional cerebral perfusion alterations in patients with mild cognitive impairment and Alzheimer disease using dynamic susceptibility contrast MRI. Acad Radiol. 2013;20(6):705-11. doi:10.1016/j.acra.2013.01.020.

65. Chen Z, Ma L. Grey matter volume changes over the whole brain in amyotrophic lateral sclerosis: A voxel-wise meta-analysis of voxel based morphometry studies. Amyotroph Lateral Scler. 2010;11(6):549-54. doi:10.3109/17482968.2010.516265.

66. Radua J, Mataix-Cols D. Voxel-wise meta-analysis of grey matter changes in obsessive-compulsive disorder. Br J Psychiatry. 2009;195(5):393-402. doi:10.1192/bjp.bp.108.055046.

67. Bora E, Fornito A, Yucel M, Pantelis C. Voxelwise meta-analysis of gray matter abnormalities in bipolar disorder. Biol Psychiatr. 2010;67(11):1097-105. doi:10.1016/j.biopsych.2010.01.020. 